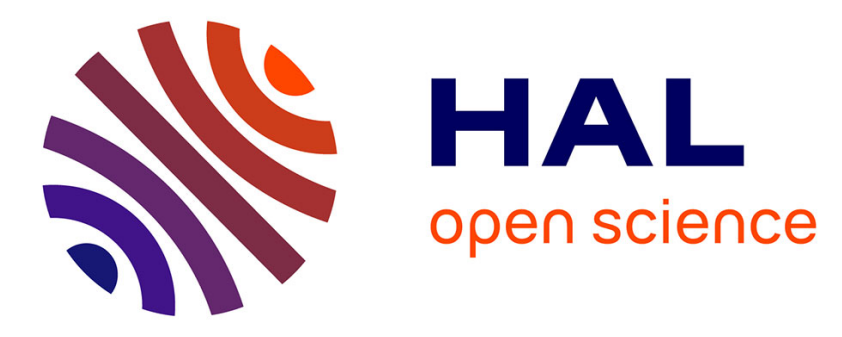

\title{
Socio-technical Design of an App for Migrants Rescue Operations
}

\author{
Stefano Valtolina, Barbara Rita Barricelli, Alessandro Rizzi, Sabrina \\ Menghini, Ascanio Ciriaci
}

\section{To cite this version:}

Stefano Valtolina, Barbara Rita Barricelli, Alessandro Rizzi, Sabrina Menghini, Ascanio Ciriaci. Sociotechnical Design of an App for Migrants Rescue Operations. 16th IFIP Conference on HumanComputer Interaction (INTERACT), Sep 2017, Bombay, India. pp.140-147, 10.1007/978-3-31992081-8_14. hal-01821417

\section{HAL Id: hal-01821417 \\ https://hal.inria.fr/hal-01821417}

Submitted on 22 Jun 2018

HAL is a multi-disciplinary open access archive for the deposit and dissemination of scientific research documents, whether they are published or not. The documents may come from teaching and research institutions in France or abroad, or from public or private research centers.
L'archive ouverte pluridisciplinaire HAL, est destinée au dépôt et à la diffusion de documents scientifiques de niveau recherche, publiés ou non, émanant des établissements d'enseignement et de recherche français ou étrangers, des laboratoires publics ou privés.

\section{(c)(1)}

Distributed under a Creative Commons Attribution| 4.0 International License 


\title{
Socio-Technical Design of an App for Migrants Rescue Operations
}

\author{
Stefano Valtolina ${ }^{1}$, Barbara Rita Barricelli ${ }^{1}$, Alessandro Rizzi ${ }^{1}$, \\ Sabrina Menghini ${ }^{2}$, and Ascanio Ciriaci ${ }^{2}$ \\ ${ }^{1}$ Dept. of Computer Science, Università degli Studi di Milano, Italy \\ \{name.surname\} @unimi.it \\ ${ }^{2}$ INMM s.r.l., Italy \\ \{name. surname\} @inmm. it
}

\begin{abstract}
This position paper illustrates the research and development work done in the last years for understanding how to support domain experts in the rescue operations of migrants who attempt to reach Italian coasts via sea journeys on Mediterranean routes. The context, characterized by humanitarian, social, and organizational issues, presents complex challenges that can only be tackled with a multidisciplinary, participatory, and internationalized approach. The final system takes inspiration from other projects developed for different purposes but acting in similar context and according to similar socio-technical dimensions.
\end{abstract}

Keywords: Participatory design, Human Work Interaction Design, international development, domain experts, migration, emergency medical services.

\section{HWID for Emergency Medical Services}

In recent years, Italy is handling the difficult situation of migratory flows ending with landing on the southern coast of the Country. According to UNHCR (United Nations High Commissioner for Refugees) report [1], since the beginning of 2017, 50,275 immigrants have entered Italy from the Mediterranean. In [2] World Health Organization defines a mass casualty incident as "an event which generates more patients at one time than locally available resources can manage using routine procedures. It requires exceptional emergency arrangements and additional or extraordinary assistance". This definition is well suited to describe what happens during the rescue operations for managing immigrants 'landing when it is necessary to offer medical assistance to a number of people who often exceed what the relief structures can accommodate. These conditions make it essential to study and implement specific strategies and work plans observed by all actors involved. Assistance operations are carried out by staff specialized in various disciplines such as doctors, nurses, and paramedics. Experts from different domains are called upon to perform their profession in a complex environment and with very challenging timing and intervention modes. This means having to perform sensitive tasks in a short time, though maintaining a high level of security, efficiency, and 
reliability of performance. This leads to the need of designing and developing IT applications to support the whole rescue operations. In particular, our research and development work is framed into studying how to design the interaction of IT solutions for enabling Emergency Medical Services (EMS). EMS are defined in [3] as "[...] the ambulance services component that responds to the scene of a medical or surgical emergency, stabilizes the victim of a sudden illness or injury by providing emergency medical treatment at the scene and transports the patient to a medical facility for definitive treatment". However, the issues in this context do not relate only with medical assistance: operation workflows have to be put in place and leadership and organizational aspects have to be faced. To manage rescue operation in an efficient way means to nominate one or more managers who can successfully lead and coordinate all team members. One of the most critical actions to be taken in rescue operations is the triage, i.e. efficiently determining severities of injuries and prioritizing treatments; this action constitutes one of the most important tasks for basic life support. Furthermore, there are other crucial activities, like organizing and running specifics areas of operation for triage, treatment and transportation. Therefore, it is mandatory to take care of the specific flow of information between the operation managers and the team members. Such a research context can be clearly seen as framed into Human Work Interaction Design (HWID) [4, 5, 6, 7], a lightweight version of Cognitive Work Analysis, addressing the concept of Work in Human-Computer Interaction. The background and experience we bring in the field is twofold. On one hand, INMM - In Manibus Meis - is a registered supplier to NATO and is responsible for providing medical information support systems for first responders and military rescuers, medical control systems for first aid rescue teams, supporting systematic collaborative to emergencies management. On the other hand, the members of MIPS (Multimedia Interaction Perception Society) Laboratory of Università degli Studi di Milano bring into play their experience in interaction design for domain experts in several application domains [8, 9, 10, 11, 12].

\section{Motivations}

The project aims at investigating how mobile digital tools used during critical and emergency activities not only facilitate the intervention on the field but also guarantee a benefit to whole related organizational structure.

The idea takes inspiration from other projects carried out in different contexts but designed around similar socio-technical principles. One example can be mKrishi@ fisheries (see Fig. 1), an app developed by Tata Consultancy Services (TCS) in collaboration with the Innovation Lab Mumbai, Central Marine Fisheries Research Institute (CMFRI), Indian National Center for Ocean Information Services (INCOIS) and Indian Council of Agricultural Research (ICAR). This app aims at providing fishers in the Maharashtra state of India with a geo-referenced map indicating to where there is likely to be many fish. Through an algorithm able to analyze satellite images, weather conditions, data of the past catch, this app can help fishers to find locations which higher concentration of fish can be reached in safety condition. 
Besides a real help to fishers, the app offers great benefits to the network of local fishery societies. For example, it was possible to register a reduction of consumption of fuel, whose distribution is under the responsibility of the fishery societies. The equal access to the information for each fisher has favored their cooperation and collaboration. Moreover, the growth in the use of mKrishi has seen and is still seeing the birth of a platform that includes services for equal fish marketing and suggestions for a more sustainable fishing procedure.

Examples like this leads us to believe that a single app used to support rescue and assistance operations in Italy can improve the whole organizational structure favoring better cooperation between all operative units and government agencies in order to enable more equal immigrants' treatment and distribution among all European countries.
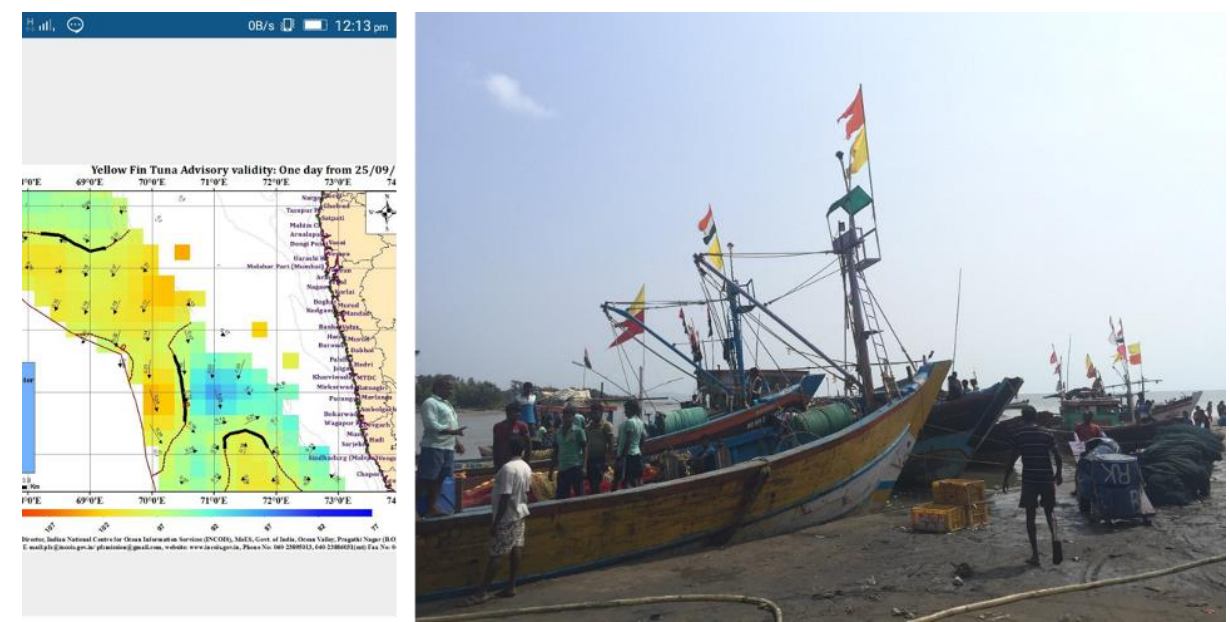

Fig. 1. A screenshot of the mKrishti app with indications to where finding fish (left) and Fisheries landing center in Alibaug village (right). Screenshots courtesy of TCS, Innovation Lab Mumbai, ICAR, CMFRI Mumbai, and INCOIS.

\section{Challenges}

In designing and developing IT solutions to be used in such a critical context, we identified six main challenges:

1. Time and resources management: the applications need to support the rescuers in gathering medical data and in managing the operation as a whole as quickly as possible but keeping a high-level quality of the actions.

2. Clinical risk reduction: electronic guides are provided to avoid incomplete and incorrect medical data collection in stressful situations, which could impair the final outcome exposing patients to possibility of mistakes along the rescue chain.

3. Human rights preservation: the entire workflow needs to be addressed efficiently and in reasonable time but always paying attention to not overcome human rights 
and dignity in the process. Specifically, discriminations on any ground have to be avoided.

4. Privacy preservation: medical data have to be managed in compliance with law requirements. This means to collect, store, protect and use all gathered data in conformance with the requirements of legislation and regulations, both on a National and EU level [13].

5. Internationalization: there are two different aspects of the context that require an internationalized approach. Firstly, the migratory wave is characterized by a multiplicity of different nationalities. To enable the collection of medical data and informed consent, and to efficiently and effectively deploy medical care, any IT application has to be designed and developed in more than one language. Secondly, it is desirable to trigger an information exchange process in order to facilitate the transmission and analysis of the data between European countries.

6. Security: information security controls had to be implemented to protect databases against compromises of their confidentiality, integrity and availability.

\section{Our Contribution}

INMM in collaboration with researchers of Università degli Studi di Milano have designed and developed ITHEALTH (International Traveller Health Surveillance System), a digital tool that through a tablet device provides rescuers with a set of functionalities for gathering patients' medical data and for managing and coordinating rescue operations. ITHEALTH allows rescuers to assign a bracelet to the patient tagged with a unique alphanumeric code (manually, by reading an NFC chip or scanning a QR Code), and then screening is assisted by the system in use at entry points (seaports and on board of ships) and along with transfer and relocation of migrants and refuges.

Each authorised caregiver is assigned a portable device (tablet or smartphone); each migrant is assigned a medical tag. All personal data is securely encrypted in it, the medical tag is worn by the migrant as his/her right to access to health care.

The resulting electronic health records (EHRs) are automatically and securely stored locally both in the ITHEALTH storage system and on the medical tag. Only authorised personnel who have access credentials to the system can see the data, thus physically the data is sent and visible only on authorized devices: laptop or desktop computer.

When any connection is available data can be transferred to a server, installed on a laptop computer, normally placed at an operating centre and/or at the Hospital.

There are additional features included:

In case of arrival from an epidemiological 'area of risk', the system, updated with previous preloaded information, matches data and instruct caregivers to deepen screening while alerting of a possible threat. In case a disease is confirmed, the caregiver is assisted to command the prompt evacuation by adopting the EVAC protocol, hospitalisation ad or isolation of the case, sending an alarm to the main Institution that there may be the risk of a public health threat to monitor. 
The workflow implemented in ITHEALTH follows official protocols and standard procedures, so that screening is guided through unified protocols, throughout the whole chain of care.

The digitalization of such protocols allows to face the first two challenges mentioned in the previous Section - i.e. time and resource management and clinical risk reduction. The quality of the workflow is guaranteed by the implementation of standard protocols, whereas the digital processes enable a quick data collection, management, and delivery. When a rescuer collects data, an informed consent is shown to the migrant to be signed. This page is translated in a set of languages and offers information about the reasons behind the data acquisition process.

Fig. 2 depicts a set of screenshots that present some steps of the medical acquisition process of ITHEALTH. Fig. 2(a) requires to insert or a new immigrant or to load data previously registered in an electronic bracelet assigned to an immigrant. The forms in Fig. 2 (c) and (d) are used for collecting immigrant's status and biographical data and medical data respectively. In this latter case, data concern heart rate, oxygen saturation and body temperature. Finally, Fig. 2 (d) requires immigrant signs the informed consent about the gathered data. This page is translated into the immigrant's language if it is possible. 
(a)

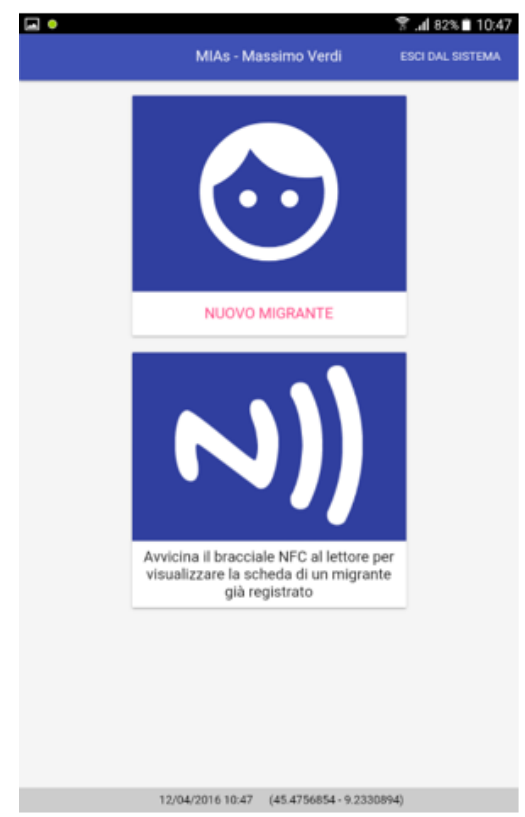

(c) (b)

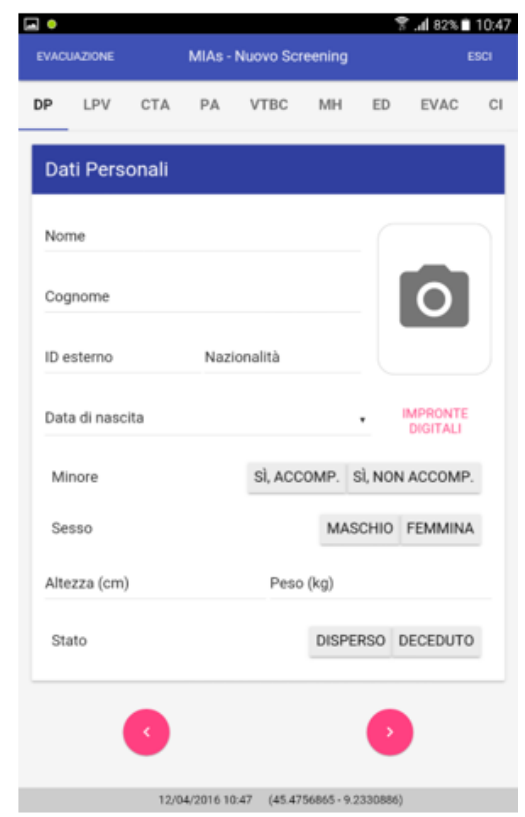

(d)
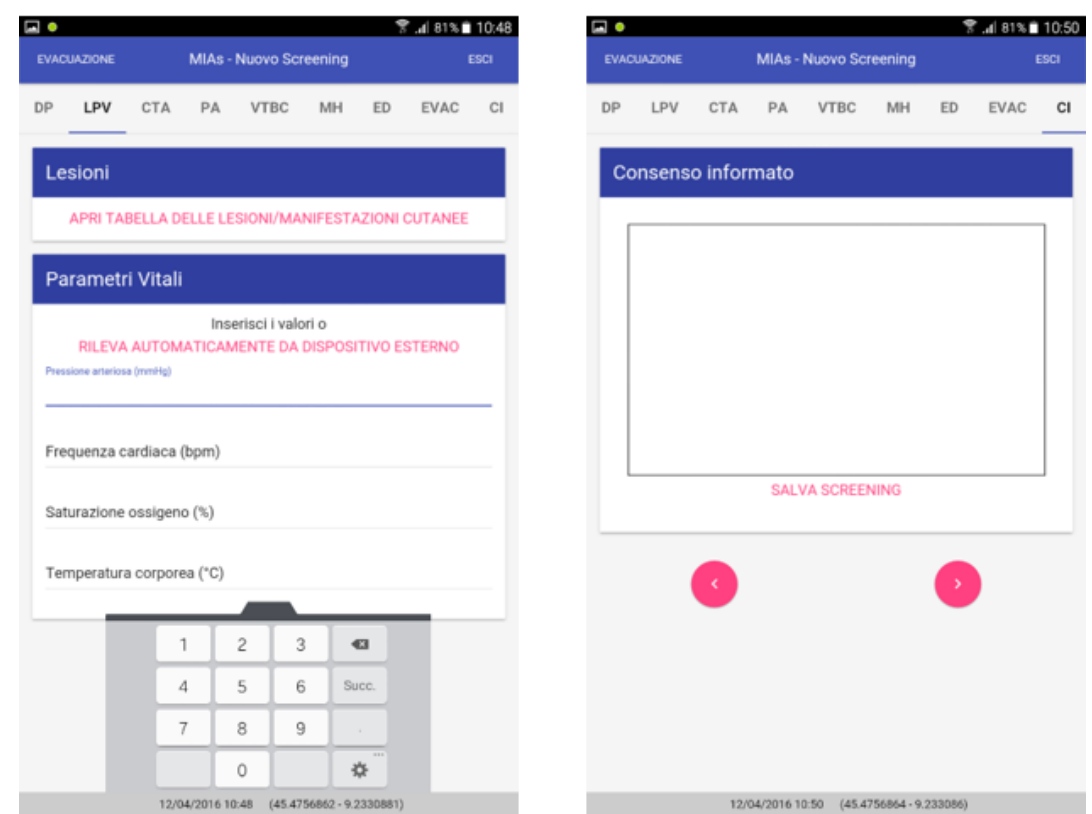

\section{Consenso informato}

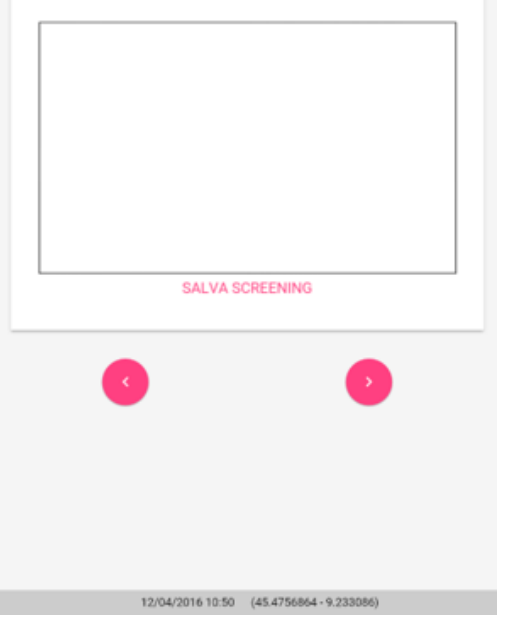

Fig. 2. Screenshots of some pages of the ITHEALTH medical data acquisition process 
When an intervention is completed, the resulting EHRs are automatically and securely stored locally both in the ITHEALTH storage system and on the medical TAG. When communications are available, the rescuer can send the EHRs and the additional information through any communication channel available (recently radio communications have been exploited too). The server keeps a database that securely stores all that has been done during the operations, enabling the creation of reporting to use for coordinating different rescues and for enabling the cooperation between various intervention agencies. About human rights and privacy presentation, EHR systems need to manage new and additional safeguards to address the fundamental conflicts and dangers of exchanging information in an electronic environment [13]. As said in the previous section, any IT application has to be designed and developed in more than one language. This is to mitigate misunderstandings between the rescuer and the immigrant about data processing and collection purposes could block or slow down the information transmission process. To face off this possible communication gaps ITHEALTH tries to report medial information by using appropriate images and very simple interfaces that can be understood even if the migrant does not speak one of the languages known by the rescuers.

Fig. 3 presents another screenshot used for reporting possible pain, fractures, burns, wounds, skin manifestations or amputation. By sketching on the figure, the rescuers can annotate where the problem is located by using a color code to specify the type of problem. This visual strategy is very useful and simple to use for the rescuer and very understandable for the immigrant too.

Finally, to deal with the second aspect concerning international issues we designed an information exchange protocol among the parties in order to: (i) set forth the information to be exchanged, the operational procedures to be followed, and the security mechanisms and other safeguards to be maintained; (ii) and set out the ways that such exchange of the particular information would be consistent with the purposes. To this aim, ITHEALTH provides modules that allow coordinators of the involved teams to follow remote rescue operations, giving orders, guiding the actions of the individual rescuer, recording data about injured, and setting up coordination tasks. In order to evaluate ITHEALTH we carried out several tests in different scenarios for testing how the tool can support rescuers during their actions and other rescue operations in order to decrease their workload while accomplishing several unusual tasks in parallel and under time pressure. 


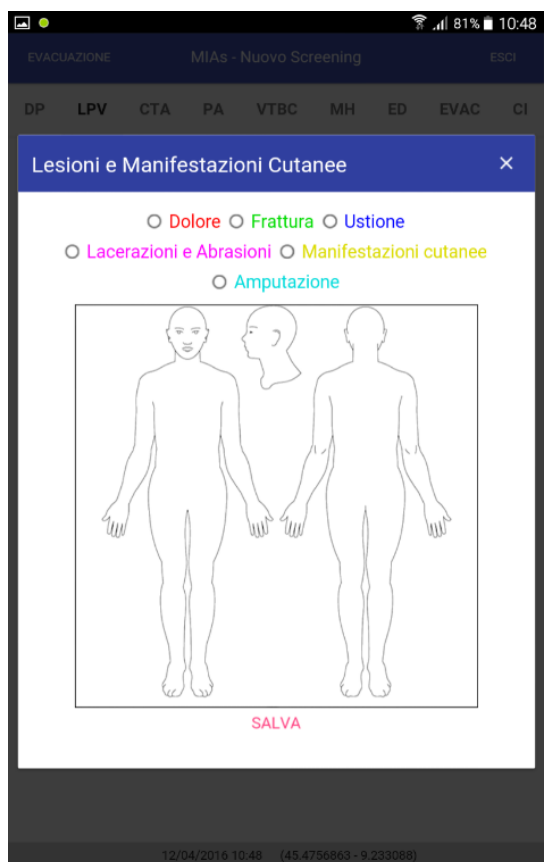

Fig. 3. A screenshot that depicts how medical data are gathered in a visual and intuitive way.

\section{References}

1. The Mediterranean Refugees/Migrants Data Portal UNHCR. https://data2.unhcr.org/en/situations/mediterranean. Accessed 24 May 2017.

2. WHO (2007). Mass casualty management systems strategies and guidelines for building health sector capacity. http://www.who.int/hac/techguidance/MCM_guidelines_inside_final.pdf Accessed 24 May 2017

3. World Health Organization (2008). Emergency medical services systems in the European Union. http://www.euro.who.int/_data/assets/pdf_file/0003/114564/E92039.pdf Accessed 24 May 2017

4. Clemmensen, T., Campos, P., Ørngreen, R., Pejtersen, A.M., Wong W. (Eds.): Human Work Interaction Design: Designing for Human Work. Springer US (2006).

5. Katre, D., Ørngreen, R., Yammiyavar, P., Clemmensen, T. (Eds.): Human Work Interaction Design: Usability in Social, Cultural and Organizational Contexts. Springer-Verlag Berlin Heidelberg (2010).

6. Campos, P., Clemmensen, T., Abdelnour Nocera, J., Katre, D., Lopes, A., Ørngreen, R. (Eds.): Human Work Interaction Design. Work Analysis and HCI. Springer-Verlag Berlin Heidelberg (2013).

7. Abdelnour Nocera, J., Barricelli, B.R., Lopes, A., Campos, P., Clemmensen T. (Eds.): Human Work Interaction Design. Work Analysis and Interaction Design Methods for Pervasive and Smart Workplaces. Springer International Publishing (2015). 
8. Barricelli, B.R., Fischer, G., Mørch, A., Piccinno, A., Valtolina, S. Cultures of participation in the digital age: Coping with information, participation, and collaboration overload (2015) LNCS 9083, pp. 271-275. DOI: 10.1007/978-3-319-18425-8_28

9. Barricelli, B.R., Gheitasy, A., Mørch, A., Piccinno, A., Valtolina, S. Culture of participation in the digital age: Social computing for learning, working, and living (2014) Proc. AVI, pp. 387-390. DOI: 10.1145/2598153.2602223

10. Barricelli, B.R., Devis, Y., Abdelnour-Nocera, J., Wilson, J., Moore, J. MANTRA: Mobile anticoagulant therapy management (2013) Proc. PervasiveHealth 2013, pp. 278-281. DOI: 10.4108/icst.pervasivehealth.2013.252096

11. Zhu, L., Vaghi, I., Barricelli, B.R. A meta-reflective wiki for collaborative design (2011) Proc. WikiSym 2011, pp. 53-62. DOI: 10.1145/2038558.2038569

12. Gianni, G.B., Marzullo, M., Valtolina, S., Barricelli, B.R., Bortolotto, S., Favino, P., Garzulino, A., Simonelli, R. An ecosystem of tools and methods for archeological research (2012) Proc. VSMM 2012, pp. 133-140. DOI: 10.1109/VSMM.2012.6365917

13. Article 29 Data Protection Working Party (2016), supra note 130, at 11-12. http://ec.europa.eu/newsroom/just/item-detail.cfm?item_id=50083 Accessed 24 May 2017 